\title{
An IEEE 802.15.4 Based Adaptive Communication Protocol in Wireless Sensor Network: Application to Monitoring the Elderly at Home
}

\author{
Juan Lu',2, Adrien Van Den Bossche ${ }^{1,3}$, Eric Campo ${ }^{1,2}$ \\ ${ }^{1}$ Univ de Toulouse, Toulouse, France \\ ${ }^{2}$ CNRS, LAAS, Toulouse, France \\ ${ }^{3}$ CNRS, IRIT, Toulouse, France \\ Email: juan.lu@univ-tlse2.fr, vandenbo@univ-tlse2.fr, eric.campo@univ-tlse2.fr
}

Received 21 July 2014; revised 20 August 2014; accepted 19 September 2014

Copyright (C) 2014 by authors and Scientific Research Publishing Inc.

This work is licensed under the Creative Commons Attribution International License (CC BY). http://creativecommons.org/licenses/by/4.0/

\section{(c) (i) Open Access}

\begin{abstract}
Monitoring behaviour of the elderly and the disabled living alone has become a major public health problem in our modern societies. Among the various scientific aspects involved in the home monitoring field, we are interested in the study and the proposal of a solution allowing distributed sensor nodes to communicate with each other in an optimal way adapted to the specific application constraints. More precisely, we want to build a wireless network that consists of several short range sensor nodes exchanging data between them according to a communication protocol at MAC (Medium Access Control) level. This protocol must be able to optimize energy consumption, transmission time and loss of information. To achieve this objective, we have analyzed the advantages and the limitations of WSN (Wireless Sensor Network) technologies and communication protocols currently used in relation to the requirements of our application. Then we proposed a deterministic, adaptive and energy saving medium access method based on the IEEE 802.15.4 physical layer and a mesh topology. It ensures the message delivery time with strongly limited collision risk due to the spatial reuse of medium in the two-hop neighbourhood. This proposal was characterized by modelling and simulation using OPNET network simulator. Finally we implemented the proposed mechanisms on hardware devices and deployed a sensors network in real situation to verify the accuracy of the model and evaluate the proposal according to different test configurations.
\end{abstract}

\section{Keywords}

Wireless Sensor Network, Medium Access Control, Quality of Service, Energy Saving, Mesh

How to cite this paper: Lu, J., Van Den Bossche, A. and Campo, E. (2014) An IEEE 802.15.4 Based Adaptive Communication Protocol in Wireless Sensor Network: Application to Monitoring the Elderly at Home. Wireless Sensor Network, 6, $192-204$. http://dx.doi.org/10.4236/wsn.2014.69019 


\section{Introduction}

Nowadays the aging population is constantly increasing and many countries are now facing an urgent requirement to provide appropriate home environment solutions for their citizens [1] [2]. These individuals attach a great importance to the autonomy that allows them to live mostly at home, and in their immediate environment, providing them freedom and a better quality of life. But, in the case of an accident such as a fall, faintness..., that autonomy can quickly turn into dependence. To supply solutions, some people use systems embedded on their body, such as physiological sensors or fall sensors [3]-[5]. These devices are intrusive and limitations become apparent due to the fact that the patient is often unable to use an alert system because either he is not wearing his equipment or, if he suddenly feels unwell, is unable to perform the alert activation gesture.

The solution we consider is to instrument the environment of the person. Indeed, by monitoring the main environmental characteristics of their living space, it seems to be possible to get a lifestyle pattern of the person [6]-[8]. For example, measuring temperature, humidity, luminosity, noise levels, presence..., in many strategic areas at home can provide useful data to interpret a physical activity in space and time. Data processing will determine circadian activity rhythms of the person and so will contribute to detect unusual situations and emergency cases. Generally, the challenge is to propose a suitable sensor network that allows uninterrupted data transmission in a bounded time.

Within this context, the objective of this work is to modelize and implement a complete heterogeneous sensor network allowing the measurement and the transmission of short-range data collected by the environmental sensors. The planned network will be deployed in a house or even building and transmit alert messages caused by a malfunction of environmental parameters via a continuous monitoring. So a limited scale, up to 50 nodes, seems to be sufficient for a home monitoring application. These nodes exchange data between them according to a communication protocol and, obviously, the following performance criteria are crucial to an indoor monitoring application: 1) Different QoS capacities should be provided for message delivery, especially when some timesensitive messages are zero tolerance for packet loss. 2) Power consumption is a fundamental concern for the battery constrained sensor nodes. A long lifetime network, several months or even years, is eagerly expected. 3) The network should be self-organizing, quickly deployed and robust against link failure or link establishment. For example, the failure of critical nodes can lead to the entire network failure and harm the safety of people monitored by this network.

Our work focuses on MAC layer to address the above constraints. A mesh WSN is considered because it is more flexible and robust than a star or a tree topology. However, how to construct this topology and how to provide the QoS are some issues in a mesh WSN. The avoidance of collisions between 2-hop neighbours is another goal because there is scarcely interference at distance of more than 2 hops [9]. Also, as the largest energy consumption of the nodes is due to the time spent in the receive state [10], so time slot allocation in a mesh topology is an important task to strictly limit non efficient transceiver activities such as idle listening.

This paper aims to present a beacon-enabled MAC over IEEE 802.15.4 PHY which supports mesh topology and enables guaranteed service with low energy consumption. The paper is organized as follows: in Section 2 some related works are presented. In Section 3 the proposed MAC protocol is fully described. Section 4 provides simulation results and Section 5 presents the results obtained through practical measurements in a real environment. The last section concludes the paper.

\section{Related Works}

Several standards for low-power, low-cost and short-range network have been investigated. Among them, IEEE 802.15.4 is considered as a promising way in terms of energy saving and guaranteed medium access. Many other main standards such as ZigBee, IEEE 802.15.5 and 6LoWPAN are all based on IEEE 802.15.4 PHY/MAC or backwards compatible with this standard. Therefore, we consider IEEE 802.15.4 as a starting point for our work. In part 2.1, IEEE 802.15.4 standard is briefly presented. In part 2.2, previous scientific works including classical WSN MAC protocols and recent works based on IEEE 802.15.4, have been studied and analyzed. The focus is mainly on the metrics: QoS, energy saving and mesh topology. 


\subsection{IEEE 802.15.4 Standard}

IEEE 802.15.4 [11] is a well-known standard for Low-Power and Low-Rate Wireless Personal Area Networks (LP-LR-WLAN). Several physical layers (PHYs) are proposed; the main and widely used 802.15.4 PHY operates in the $2.4 \mathrm{GHz}$ radio band and enables transmissions at $250 \mathrm{kbps}$ in a $30 \mathrm{~m}$ indoor range. The protocol supports beacon and non beacon mode. More precisely, in beacon mode, it is possible to achieve variable duty cycles (from $100 \%$ down to $0.006 \%$ ), which is particularly interesting for our application where energy constraint and network lifetime are concerned. In addition, the beacon mode has an attractive feature for time-sensitive applications as QoS properties are available with GTS (Guaranteed Time Slot) mechanism. On the other side, the non beacon mode that has the advantage of a lower complexity and a higher scalability as compared with beacon mode, does not provide any of those features. Thus, we focus our development on the beacon mode of this standard.

IEEE 802.15.4 may operate in two topologies: a star topology or a peer-to-peer topology. Peer-to-peer topology allows a more complex network to be implemented such as cluster-tree topology. However, how to construct a mesh networking topology as required by our application is missing in the standard. In fact, while the current standard supports multi-hop networking using peer-to-peer topology, it restricts its use to a non beacon mode. This contradiction makes the interesting advantages, such as GTS and energy saving, disappear, especially on router nodes.

\subsection{MAC Protocols for WSN}

Some classical WSN MAC protocols such as S-MAC [12] and ContikiMAC [13] and their optimized works such as T-MAC [14] and X-MAC [15] are based on the sleep-wake cycle to reduce energy consumption of WSN. However, they all access the medium by CSMA (Carrier Sense Multiple Access) mechanism that provides a best-effort service. So the transmission of time-sensitive messages may not be guaranteed.

ZigBee specifications [16] clear the ambiguities of IEEE 802.15.4 in a cluster-tree topology. The centralized PAN (Personal Area Network) coordinator calculates and assigns a beacon transmission offset for each node when it wants to associate the PAN. Therefore, the network scalability and flexibility are both limited. The direct communications between neighbours are not possible.

Anis Koubâa also focuses his work in the field of cluster-tree topology. In TDBS [17] [18], the requirement of different BI (Beacon Interval) and SD (Superframe Duration) for each node is calculated in advance. However, this weakens the flexibility and robustness as well as restricts the scalability of network.

Another example has been proposed in OCARI project [19] [20]. A PAN coordinator is the destination of all association requests and allows a beacon slot for each associated node. The main drawback of this solution is the lack of flexibility, especially regarding a changing topology, for example due to the inconstancy of wireless medium.

P. S. Muthukumaran proposed MeshMAC protocol [21]. This protocol enables mesh networking over the beacon mode through a distributed SDS (Superframe Duration Scheduling) strategy in which each node calculates its schedule to transmit beacons based only on locally available information. The limitations of MeshMAC are: it imposes very low duty cycles for a large scale network so that the end-to-end delay may be extended; the beacon transmission offset is difficult to choose if the topology changes.

B. Carballido Villaverde proposed DBOP MAC protocol [22] [23]. It creates a BOP (Beacon Only Period) where beacons are transmitted at different time slots among neighbours and neighbours' neighbours. However, DBOP introduces an overhead into the IEEE 802.15.4 MAC standard. Another drawback is the inefficient management of BOP length. In addition, our MAC protocol was proposed before this work and we have continued to work on it until a prototype has been totally implemented.

Hence, we consider that all the current standards and protocols cannot satisfy all our application criteria. Many problems such as beacon collision, dynamic timeslot allocation, energy saving on router nodes, determinism medium access in a mesh topology, etc., have not been solved yet. This makes urgent requirement of new MAC protocols.

\section{ADCF MAC Protocol}

This part presents an original MAC protocol named as ADCF (Adaptive and Distributed Collision Free). The 
proposed protocol aims to improve robustness and flexibility of IEEE 802.15.4 MAC, which means the capacity of self-organization and auto-reparation. Simultaneously, ADCF should enable energy efficiency and guaranteed slots negotiation [24] [25]. Before showing the details, some assumptions should be highlighted: 1) the main contribution of ADCF concerns the router nodes. We have considered an ad-hoc approach: all the considered nodes have the capacity to be both sensor and router, even if classical sensors (Reduced Function Devices) may be associated to the routers, but this last point is out of the scope of this paper. 2) To ensure full determinism in the build of the topology, nodes addresses must be preliminary set during installation.

\subsection{Overview of ADCF}

ADCF is based on the IEEE 802.15.4 2.4 GHz DSSS physical layer and classical superframe structure. On the one hand, ADCF proposes a distributed beacon scheduling mechanism in a beacon only period which spatially reuses the timeslots over 2-hop. On the other hand, the contribution of ADCF lies in a data slot allocation mechanism, which makes GTS possible in a mesh topology.

Generally, the nodes can switch between two stages: initialization stage and working stage. In initialization stage, which is not using the superframe structure, the nodes access medium by unslotted CSMA/CA mechanism. In working stage, the time is divided into superframe and each superframe includes three parts: BOP, Active Period, Inactive Period\}.

As shown in Figure 1, BOP is organized by CFBS (Collision Free Beacon Slot). Each node has a 2-hop collision-free beacon slot in BOP. Active period is divided into 16 equally sized slots as a classical IEEE 802.15.4 superframe. It starts with the CAP (Contention Access Period) where medium accesses are done by using slotted CSMA/CA mechanism. It ends with CFP (Contention Free Period) where medium accesses are done by using an original mechanism that provides GTS-equivalent for the mesh topology. This original mechanism is based on the CFDS (Collision Free Data Slot). After active period, the optional inactive period allows all the nodes to go to sleep mode for energy saving.

In a mesh network, the node that uses the first CFBS of the superframe is called as initiator. Initiator is not a supernode as each node in the network may be selected as initiator; initiator is not a supernode as it only has a partial knowledge of the 2-hop neighbourhood, like the other nodes; initiator is not a supernode as the network could work properly when initiator fails; initiator is not a supernode as it has not any central role such as a classical IEEE 802.15.4 PAN-Coordinator.

In the rest of this part, CFBS, CFDS mechanisms as well as a smart repair protocol will be presented.

\subsection{CFBS Mechanism}

The beacon frame is very important as it announces the presence of the node and the presence of its 1-hop neighbours; it maintains network synchronization and is used to request/reply neighbour data slot negotiations, i.e. CFDS. The nodes far away more than 2 hops could reuse the same CFBS to enhance channel reutilization. In other words, nodes must determine their CFBSs by taking into account CFBSs used by their 2-hop neighbourhood with a certain priority.

To determine priority, three parameters of the node are employed: 1) ND (Neighbour Density) indicates the number of neighbours within 2 hops, including the node itself. The node with the higher ND is usually in the

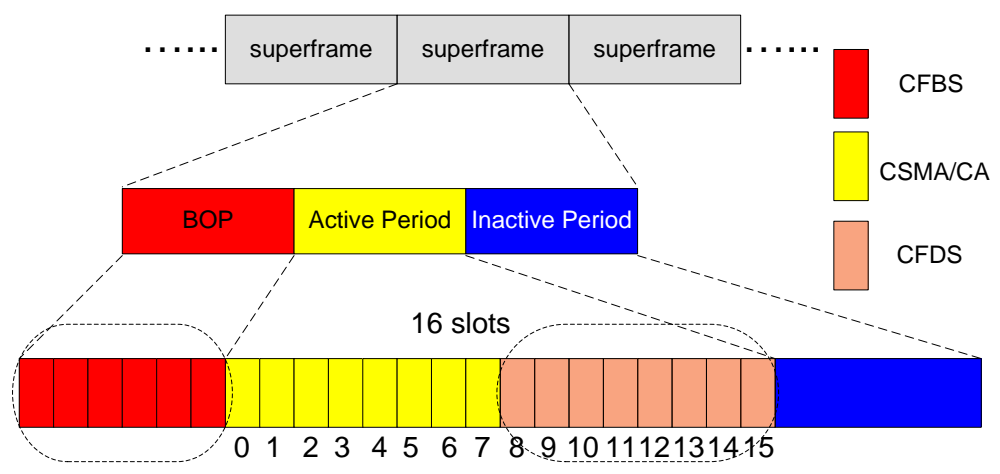

Figure 1. ADCF superframe structure. 
center zone of a network and has a faster awareness to the topological changes. 2) NE (Neighbour Energy) indicates the residual energy level of the node and has the profound effect to the network. 3) NA (Neighbour Address) means the 16-bit address of the node and is unique for each node. The node with higher ND, higher NE and smaller NA has higher priority. The comparison order is ND, NE and then NA.

The node with the highest priority in the network is selected as the initiator. It specifies the beginning of BOP and measures the length of BOP (BOPL). The initiator's ND is defined as $\mathrm{D}_{\max }$ and equals the length of BOP.

When a new node joins the network, it will firstly listen to the channel for a fixed period. Depending on the received beacons during listening, the new node will send its own beacon by different mechanisms. Each node broadcasts its beacon within 1-hop and records direct neighbours' in its NT (Neighbour Table). Therefore, all the 2-hop neighbours' information is obtained by this new node. The interesting information in a beacon includes ND, NE, NA, etc.

The node with the higher priority could choose its beacon slot earlier. Obviously, initiator occupies the first CFBS and its information is broadcasted without the hop limitation. Then other nodes, according to their priorities, can take the slots that are not used by their 2-hop neighbours and store the slot number in their NT. As each node only maintains a 2-hop NT, the nodes may choose CFBS synchronously and therefore quickly. With BOPL, each node can calculate the beginning of a superframe by the time of a received neighbour beacon and its slot number. At this moment, the node synchronizes with its neighbours and can schedule its own superframe. We can say that the node is in working stage now.

More details of CFBS mechanism have been published in [24], including beacon frame format, theoretical analysis and simulation study of protocol cost such as convergence time and the incurred message overhead.

\subsection{CFDS Mechanism}

For the time-bounded traffic or the traffic of zero-tolerance packet loss, CFDS enables these traffics to be sent in some dedicated slots. Medium access can be done directly, without backoff delays or medium sensing. This aspect is very useful in our application.

Thanks to CFBS, the CFDS negotiations can be achieved between source node and destination node using beacon exchanges. Since the beacons are broadcasted with guarantee through the CFBS, the CFDS allocation process can be time-bounded, unlike the GTS_request process of IEEE 802.15.4 that uses CSMA/CA. Similarly, nodes must determine their own CFDSs by taking into account CFDSs used by their 2-hop neighbourhoods.

For the source node, CFDS allocation is triggered by the upper layer, which contains the destination address and the length of requested CFDS. By setting flags of beacons, source node broadcasts this request to all its neighbours. When a source node receives a beacon from a destination node with the valid CFDS slot number and CFDS length, it will update the NT and broadcast the allocated slot in the next beacon. The new time schedule is also calculated in order to achieve this direct collision-free transmission. As there is no extra overhead for the CFDS negotiation, source node can continue requesting until the destination node finds the valid CFDS.

So for destination node, the objective is to search the available CFDS and respond to source node. The slot number must be decided by the receiver (destination) of the traffic to avoid frame collision. Specifically, when a node receives neighbour's beacon and finds its address as the destination, it will check its NT, allocate the first available data slot (e.g. slot 8 in Figure 1) to source node and announce this allocation in the next beacon. This available CFDS should not be reused by another node in the 2-hop neighbourhood. When source node requests several CFDSs, the slot number is noted as 8 if the following CFDSs are also available. Else, destination node searches until the end of active period (slot 15). If the available CFDSs are not enough, destination node also returns to the first available CFDS and provides CFDS services as more as possible.

The CFDS deallocation is also invoked by source node of this CFDS. Source node clears up all the flags and slot number and then sends the beacon. Destination node also clears up all the CFDS information with this source node from its NT when it receives the indicated beacon. Therefore, the deallocation is complete and the CFDS is free for other nodes.

CFDS mechanism allows point-to-point bidirectional communications in the mesh topology. When a destination node wishes to reserve CFDS with the corresponding source node, it launches the same procedure as single-direction communication. However, considering the indoor application environment and the possible large overhead (a 3-hop NT), the traffic sending by CFDS has no acknowledgement. This specific point has been successfully studied on our real testbed. In addition, if no CFDS used by the node at all, it may sleep during 
these data slots for further energy saving.

\subsection{Smart Repair Protocol}

The objective of this protocol is to minimize the impact of a change of topology as much as possible. The SRP (Smart Repair Protocol) is critical in ADCF as it improves network flexibility and robustness. Generally, topology changes could be classified as two types: node join and node failure. All other complex topological changes such as network separation and network integration are all based on the two basic changes.

In reality, node failure can be caused by energy exhaustion, unexpected damage or even the poor quality of wireless link. So a node considers the failure of its neighbour node only when the neighbour's beacon loss is above a threshold. Similarly, new node is regarded as neighbour only when its beacon loss is below a threshold. In our proposal, beacon loss threshold is a predefined parameter which depends on different wireless environments during the implementation. In other words, we estimate the wireless link quality by beacon loss. Four states are defined for a link or a node connected by the link. 1) Preliminary. 2) Unconfirmed. 3) Confirmed. 4) Deleted. Therefore, node join or node failure here means the presence or disappearance of one node in its neighbours' NT. Both unconfirmed nodes and confirmed nodes are stored as 1-hop neighbours in NT. But only confirmed nodes are chosen as 1-hop neighbours when constructing beacon frame.

Asymmetric links are possible and acceptable. In ADCF, both CFBS and CFDS mechanisms are over a 2-hop NT. If two neighbours connected with an asymmetric link choose the different slot to transmit the beacon or data frame, the collision could be avoided and that's what we expect. If two neighbours connected with an asymmetric link choose the same slot, they will transmit the beacon or data frame at approximate the same time. In this situation, this asymmetric link will be abandoned and other high quality link may be utilized. The redundant mesh link of ADCF also improves the communication reliability.

Based on the above definitions, the following two parts specify different mechanisms depending on different topology change cases.

1) Node join and BOP augmentation. Except full mesh network, multiple-hop network may reuse the medium so that there are always free slots in BOP. A new node may choose its CFBS directly after the listening period and join the network immediately. So ADCF has the great advantage to adapt the topology change. For full mesh network, if a new node wants to join the network and finds BOPL is not sufficient $\left(D_{\max }>\right.$ BOPL), the new node may send its beacon by CSMA until a new initiator is designed with updated $D_{\max }$. In this process, the nodes will return to initialization stage.

2) Node failure and BOP reduction. If link failure is detected by beacon loss mechanism, neighbours will simply delete this failing node from NT. If the initiator fails, others re-select an initiator but keep their BOP with the original slots. Therefore, the network will still work without disruption. In one case $\left(D_{\max }>B O P L / 2\right)$, the nodes will also return to initialization stage for an optimal BOP.

In conclusion, our application requires an adaptive communication protocol providing QoS-guaranteed service with reasonable energy consumption in a mesh network. With ADCF, we proposed two mechanisms called CFBS and CFDS to enable efficient deterministic medium access for beacon and data. The Smart Repair Protocol may further improve the network flexibility and robustness. In the following parts, we will present the performance results obtained by the simulation and the prototyping work.

\section{Simulation Study}

To study the scope of our contribution, we use OPNET to develop a simulation model that implements the ADCF MAC. Many scenarios and metrics were simulated. For example, we investigated the protocol cost of initialization stage, the network performance with different topology change cases, and the network performance in large scale and high-density situations, etc. [25]. In this paper, we choose to present the comparison of ADCF with IEEE 802.15.4 (ZigBee version implementation [26]). An ideal IEEE 802.15.4 physical layer, indicating the channel without random packet loss, is applied for both protocols. A more practical energy model [27] is used in the simulation. A static routing mechanism achieved by prior manually adding routes is above ADCF in order to simulate application traffics over the network. Buffers sizes are consistent with the prototype. In addition, the topology is a random mesh configuration. Other basic parameters are shown in the Table 1.

According to our energy model, the actual energy consumption in reception mode is larger than the energy consumption in transmission mode. Therefore, even though there is no application traffic, the active period al- 
ways keeps in receive mode, which consumes more energy. So only beacons are delivered in order to compare the protocols cost. As shown in Figure 2, it can be seen that ADCF consumes less energy, about 37\%, compared to IEEE 802.15.4 as time goes by. This is because the active period is the same for all the ADCF nodes, while IEEE 802.15.4 router nodes have two active periods. One is for the communication with parents and the other is for the communication with children. Hence IEEE 802.15.4 spends more time for idle listening. In theory, no matter what topology, if BOPL is less than the active period, ADCF always consumes less energy than the IEEE 802.15.4 standard.

As shown in Figure 3, there are 7 sources with 1-hop traffic or 3 sources with multi-hop traffic. When Packet Interarrival Time decreases from $1.0 \mathrm{~s}$ to $0.1 \mathrm{~s}$, the traffic load will increase. Therefore, End-to-end delay becomes larger. When Packet Interarrival Time is about $0.4 \mathrm{~s}$, there are radical changes caused by buffer overflow.

With the current superframe ( $2 \mathrm{~s}$ ) and active period configurations, the end-to-end delay for 1-hop traffic is about $1 \mathrm{~s}$ on average, including the time from packet generation to the scheduled data slot. ADCF has $0.06 \mathrm{~s}$ advantage than IEEE 802.15.4. If a packet arrives just before its scheduled data slot, the node can send this packet immediately. Otherwise, the node may send the packet in the next superframe. It also can be seen that ADCF saves about $25 \%$ end-to-end delay for multi-hop traffic. This is because some multi-hop traffic may be transmitted in one superframe as the same active period for all the mesh nodes. IEEE 802.15.4 works in a cluster-tree topology which may take several superframes from the source to the final destination. The average hop count is 3 , so this end-to-end delay is about $2.7 \mathrm{~s}$ for ADCF and $3.5 \mathrm{~s}$ for IEEE 802.15.4. In some other topology cases, ADCF can be even more efficient since the 802.15.4 tree can be shortened thanks to the mesh links.

In the same simulation, when the CFDS buffers are available, both ADCF and IEEE 802.15.4 keep 100\%

Table 1. Basic simulation parameters.

$\begin{array}{cc}\text { Parameter } & \text { Value } \\ \text { Scene area } & 100 \times 100 \mathrm{~m}^{-2} \\ \text { Transmission range } & 15 \mathrm{~m} \\ \text { Number of nodes } & 14 \\ \text { Superframe period } & 2 \mathrm{~s} \\ \text { Active period } & 0.245 \mathrm{~s} \\ \text { Application payload } & 100 \mathrm{bits} \\ \text { CSMA buffer } & 0.5 \mathrm{k} \text { octets } \\ \text { CFDS buffer } & 1.5 \mathrm{k} \text { octets } \\ \text { Simulation duration } & 30 \text { min } \\ \text { Simulation times } & 20\end{array}$

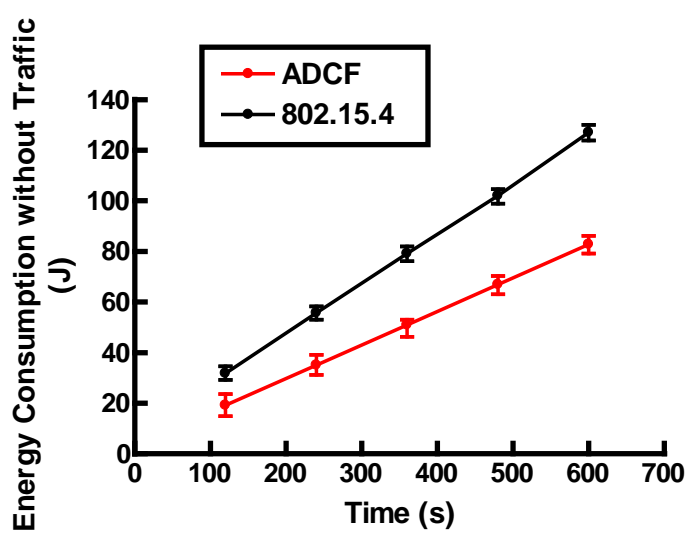

Figure 2. Energy consumption comparison. 


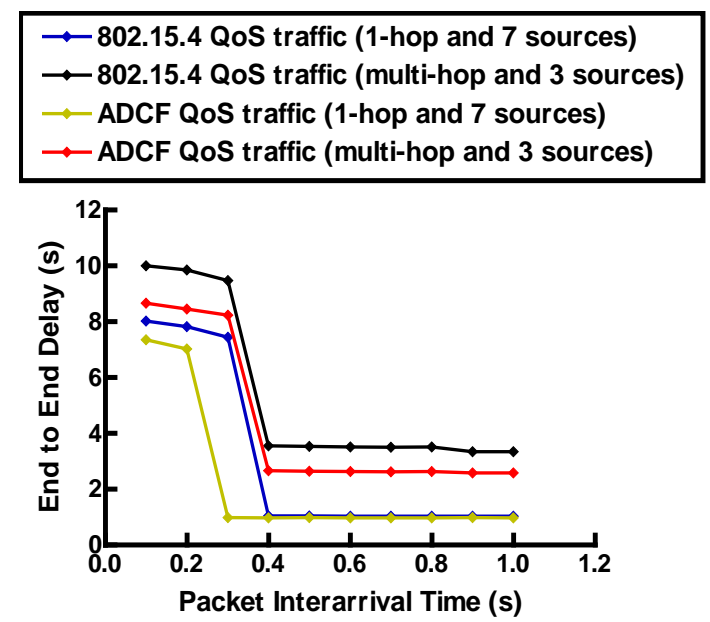

Figure 3. End-to-end delay comparison of traffic sent by CFDS.

packet success ratio, which indicates the percentage of successfully delivered packets.

By many other simulation works and results, we can conclude that ADCF satisfies our application request of delivering QoS messages with low energy consumption. Therefore, for the monitored people, environmental alerts, and remote assistant messages could be quickly sent to the destination without loss. The end-to-end delay is optimized, especially for the multi-hop alters traffic.

\section{Prototype Implementation}

Prototype implementation is a fundamental approach to verify a protocol and its performances. A platform WiNo [28] of emulation and rapid prototyping for WSN is utilized. WiNo is an open platform ready to accommodate protocols at MAC layer or network layer for sensor networks. It provides an open environment adapted to research projects, including the management of physical layer and the necessary tools to develop a full but very compact protocol stack using ANSI C-language. More accurately, a developer with WiNo can master not only the time access to the medium and the sleep-wake cycle, but also the CPU time and memory resource generally restricted by hardware in a WSN.

Currently, two types of nodes from Freescale, 13192-SARD and 1321x-SRB, are implemented with ADCF MAC. Both of them have the common features: 1) IEEE 802.15.4 2.4 GHz transceiver. 2) 8-bit MCU. 3) There are $4 \mathrm{~KB}$ of RAM and $60 \mathrm{~KB}$ of on-chip Flash. Two tools are mainly used for debugging, measuring and evaluating the protocol performance in real conditions: the date-logger console of nodes and SNA (Sensor Network Analyzer) [29]. Daintree's SNA is known as an expert tool providing comprehensive solution for IEEE 802.15.4 and ZigBee. It includes a protocol analyzer software fitted to sensor networks and a sensor network adapter hardware. The sensor network adapter hardware is a powerful node, which can capture all the packets of a network but do not participate in this network. By SNA software that includes a protocol decoder allowing user to drill down to packet, field and byte level, we can view all network nodes and interactions simultaneously, without disturbing the original network.

In part 5.1, two results obtained through practical measurements in our laboratory will be given. In part 5.2, the deployment of ADCF in a real application context using our smart home of Blagnac [30] will be presented.

\subsection{Experimental Scenarios and Results}

To build the multi-hop network, 8 nodes are gradually deployed in our laboratory. In each scenario we start each node at different time with a random MAC address. In fact, each multi-hop network is unique and has its own particularities. We do 20 times manipulations separately for the following two networks examples, as shown in Figure 4 and Figure 5. In the first deployment, 8 nodes are disposed throughout the first floor of our laboratory. And in the second deployment, 4 nodes are disposed in the first floor, 3 nodes are disposed in the ground floor, 1 node is disposed on stairway. Even though the fixed positions, network topology may change due to the wireless 


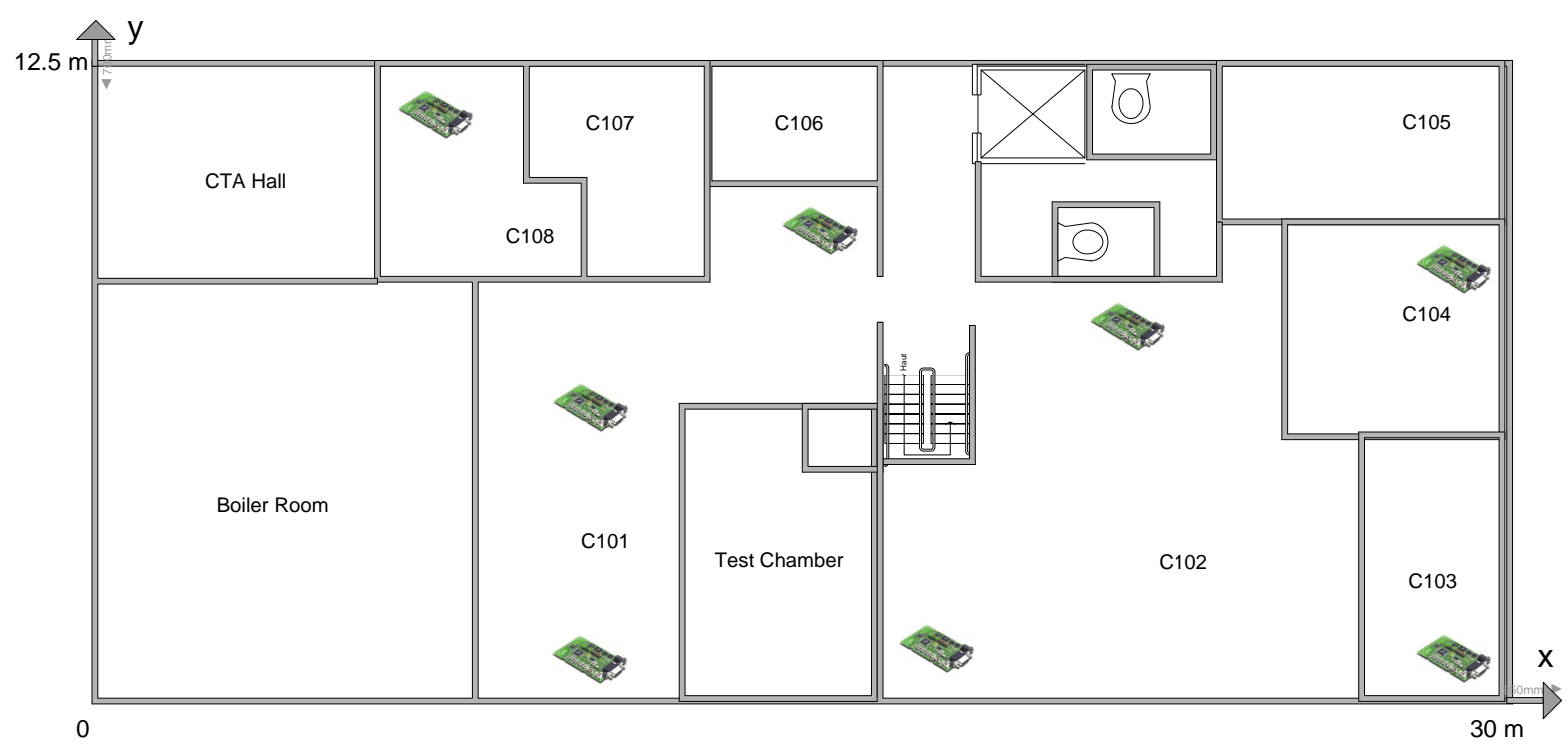

Figure 4. Network example 1.

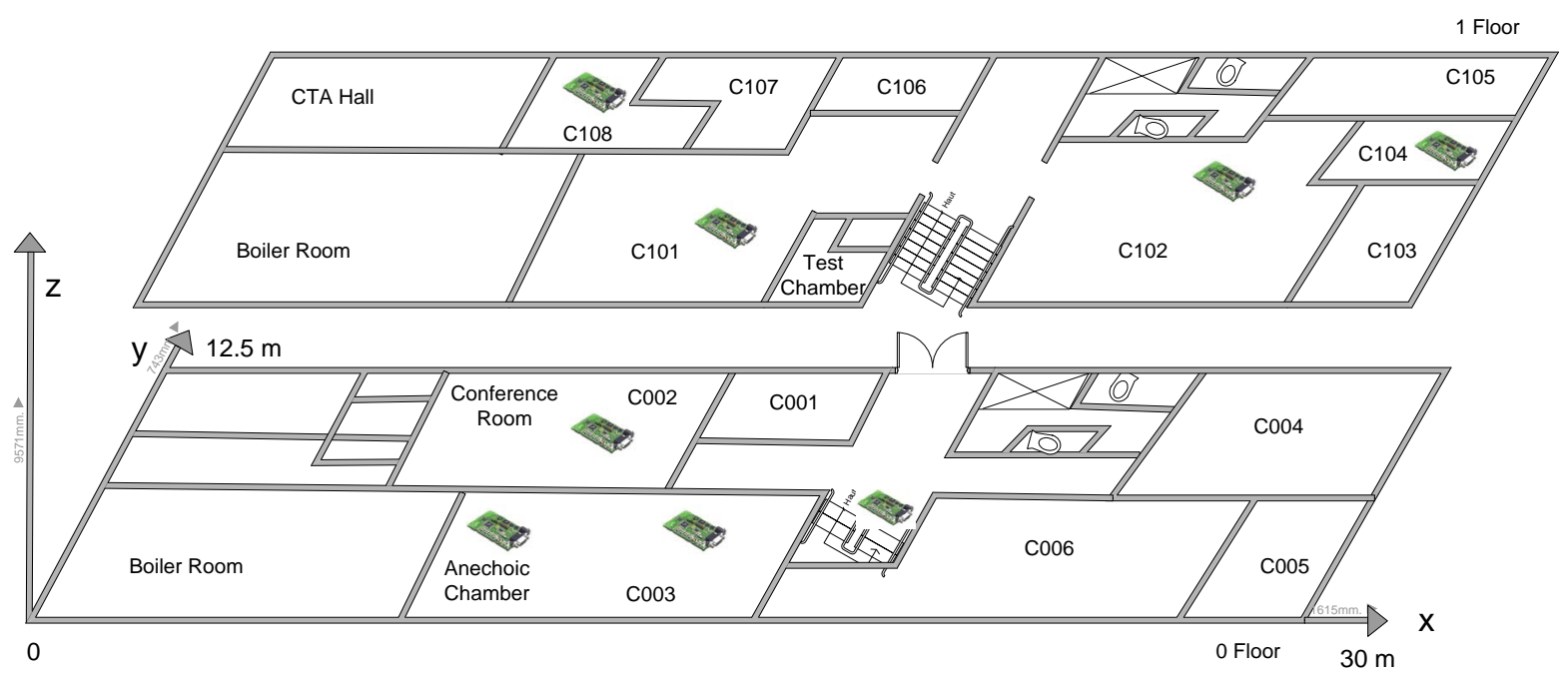

Figure 5. Network example 2.

links. Based on the SNA results, the maximum hop count is 3 in the two network examples. Some interesting results are shown in Table 2.

Here convergence time of the last node means the time duration from the start of the last node to a BOP well organized network in which each node has a collision-free beacon slot. In reality for the deployment of multi-hop topology, power-up of each node in the network is a process. At the same time, as in the mesh network each node can directly communicate with its neighbours, therefore network rebuilding may be triggered in this deployment process. We can see that this convergence time takes about 1 to 2 minutes on average. This value is acceptable considering the total network lifetime which is several days or weeks. In a normal situation where the nodes are fixed, the network topology may not be reorganized frequently. The reuse of CFBS confirms the successful achievement of the organized superframe and the synchronized multi-hop networks. In addition, this test highlights that asymmetric link occurs frequently in the real environment, but ADCF can work well without disturbing of asymmetric link problem.

\subsection{Deployment of ADCF in a Smart Home}

The smart home of Blagnac in France targets the elderly and the disabled living alone and provides them health 
Table 2. Results for multi-hop network.

\begin{tabular}{cccc}
\hline & & Network example 1 & Network example 2 \\
\hline \multirow{2}{*}{ Convergence time of the last node } & Average & $123.99 \mathrm{~s}$ & $97.74 \mathrm{~s}$ \\
& Minimum & $55.04 \mathrm{~s}$ & $42.63 \mathrm{~s}$ \\
Reuse 1 CFBS & Maximum & $252.30 \mathrm{~s}$ & $173.25 \mathrm{~s}$ \\
Reuse 2 CFBS & $19 / 20$ & $11 / 20$ \\
Asymmetric links & & $3 / 20$ & $9 / 20$ \\
\hline
\end{tabular}

and medico-social assistance at home [31]. Many types of equipment, including wired products, mainly KNXbased, have been installed and are operational in this smart home. ADCF network fills some application gaps and provides an alternative wireless solution. Our testbed is also described in a video [32].

Six ADCF nodes were deployed in the smart home. From MAC point of view, these 6 nodes form a full mesh network. The nodes are connected with various sensors corresponding to the target of our application:

- Node @106 connected with a magnetic sensor can monitor the open/closure of the refrigerator,

- Node@107 connected with an infrared sensor can detect the motion of the person under his coverage area,

- Node @108 connected with an emergency button is worn by the user and may alert in case of fall, faintness, etc.

- Node @109 connected with a sensor carpet placed near the bed can detect the getting up of the user,

- Node @10A connected with a light can be switch on/off by an order of another ADCF node,

- Finally node @105 connected with screen is a sink for collecting and displaying all the network information. On the screen, we can see the superframe structure updating each second and log files from a web page [33].

From application point of view, the data exchanges are as following (Figure 6):

- Node @106 sends a message to node @105 when the fridge is opened or closed,

- Node@107 sends a message to node @105 when the person moves under the infrared sensor,

- Node@108 sends a message to node @105 when the person presses the emergency button,

- Node@109 sends a message to node @10A when the user arrives or leaves the carpet,

- Node @10A receives messages from node @109 and switches on the lighting when the user is on the carpet. It switches off the lighting when the user leaves the carpet. When the lighting is switched on/off, node @10A sends a message to node @105 containing a return state of lighting.

- Node@105 receives messages from node@106, @107, @108, @10A and logs actions in a journal. A voice synthesis software eSpeak [34] is also available and announces the reception of information from sensors (motion, carpet, etc.). Several scenarios using the sensor information have been implemented; for example, when the sink detects the opening of the fridge for more than 10 seconds, the user can hear a voice alert.

In the initialization stage or rebuilding stage, frames are transmitted immediately without any medium access control precaution. In working stage, frames are transmitted using CFDS.

Figure 7 shows a result of the above network deployment. From the ADCF journal and application journal displayed on the screen, we can see that each node can join or leave the network freely and the rest of the network works properly. Generally, the network rebuilding time is less than $10 \mathrm{~s}$. At this moment, 6 nodes occupy 6 CFBS as they are all 1-hop neighbours. 5 CFDS are negotiated to transmit the corresponding application data. Thanks to a buzzer available on the nodes, which is activated for each data frame reception, we can verify that the bounded time is verified as expected. For example, end-to-end delay is always less than $2 \mathrm{~s}$ when the superframe is fixed as $1.5 \mathrm{~s}$. It satisfies our application requirements. In addition, the superframe with its slot allocation is totally consistent with what we proposed in Section 3.

\section{Conclusions and Perspectives}

This paper presented an original MAC protocol ADCF based on the IEEE 802.15.4 standard. This protocol was designed to build and to maintain a wireless mesh sensor network providing QoS-guaranteed medium access and energy saving solution for home monitoring application. The work was organized in three phases: protocol 

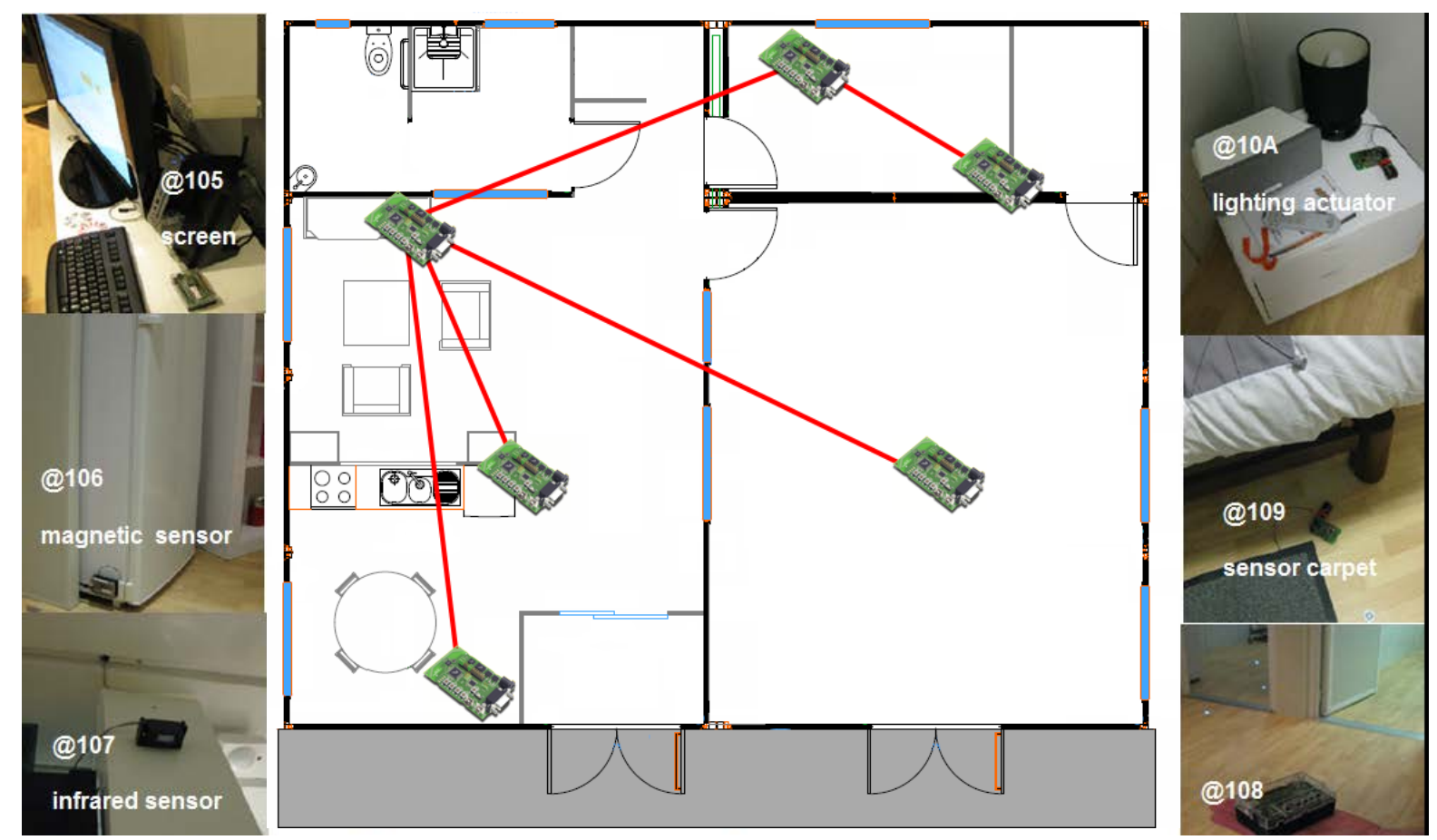

Figure 6. Six ADCF sensor nodes and the data transmission topology at application layer.

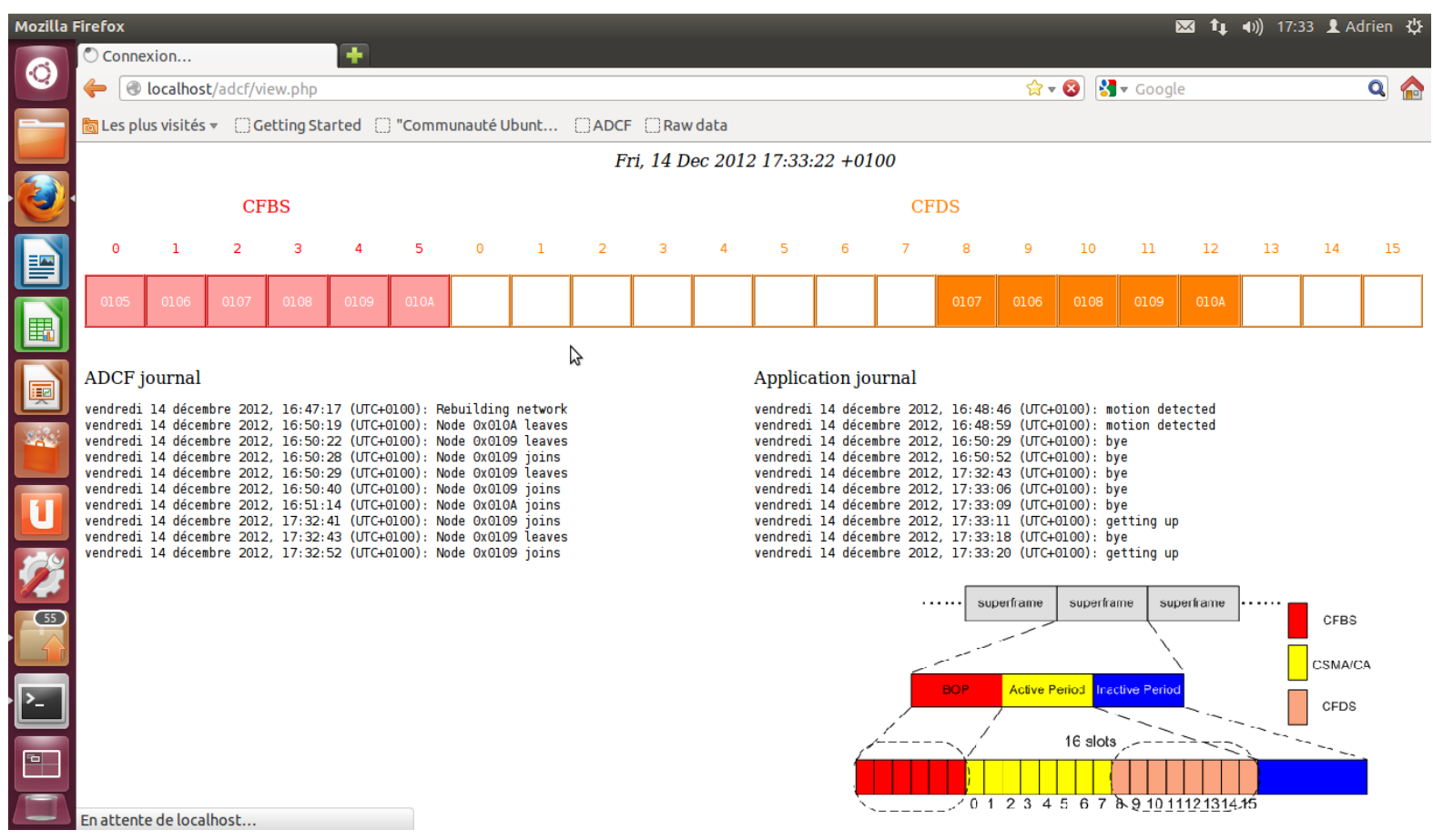

Figure 7. Example of a visual result.

presentation, implementation and results from simulation and from prototype. In conclusion, the contributions of this paper are:

1) The 2-hop CFBS and CFDS mechanisms were proposed and described. CFBS enables beacon collision to be avoided and therefore efficient multi-hop mesh network to be formed. Thanks to CFBS, CFDS enables the application data to be transmitted in a bounded time without loss. A smart repair protocol was also proposed to 
improve the flexibility and robustness of a mesh network. It’s efficient to manage BOPL according to the wireless topology changes.

2) We established a simulation model by OPNET. The simulation results show that ADCF satisfies our application requirements. For example of the specific topology, ADCF consumes less than 37\% energy than IEEE 802.15.4. At the same time, end-to-end delay and packet success ratio show similar performance compared to the standard. Many other scenarios were simulated and the results may be good reference to prototype.

3) We developed some prototypes which were not considered in other works. By the results obtained through practical measurements, we can see: a mesh network of 8 nodes could successfully be built with an organized superframe where each node can choose a CFBS by ADCF. A full mesh network of 6 nodes was deployed in the smart home. This network can always converge in about $10 \mathrm{~s}$ with the suitable parameters configuration and, thanks to the buzzers we can hear the reception of an application data on the sink in $2 \mathrm{~s}$ at most, which confirms that ADCF is verified in a real environment as expected.

In the future, we will optimize the prototype such as enabling the evaluation of energy consumption of hardware and compare the results with the simulation. Another perspective is in the framework of cross-layer design, such as reuse the 2-hop NT, to reduce routing overhead and further optimize network performances. Finally, we will test various application scenarios and invite the elderly and the disabled to use the system in the smart home.

\section{References}

[1] Fnet, T. (2006) Counting on an Aging Population. Computing in Science \& Engineering, 8, 88-96. http://dx.doi.org/10.1109/MCSE.2006.107

[2] Boulanger, J.R. and Deroussent, C. (2008) Preliminary Based Service Evaluation for Elderly People and Healthcare Professional in Residential Home Care Units. Second International Conference on the Digital Society (ICDS 08), 93101. http://dx.doi.org/10.1109/ICDS.2008.36

[3] Chen, S.-L., Lee, H.-Y., Chen, C.-A., et al. (2009) Wireless Body Sensor Network with Adaptive Low-Power Design for Biometrics and Healthcare Application. IEEE Systems Journal, 3, 398-409. http://dx.doi.org/10.1109/JSYST.2009.2032440

[4] Otal, B., Alonso, L. and Verikoukis, C. (2009) Highly Reliable Energy-Saving Mac for Wireless Body Sensor Networks in Healthcare Systems. IEEE Journal on Selected Areas in Communications, 27, 553-565. http://dx.doi.org/10.1109/JSAC.2009.090516

[5] Mamaghanian, H., Khaled, N., Atienza, D. and Vandergheynst, P. (2011) Compressed Sensing for Real-Time EnergyEfficient ECG Compression on Wireless Body Sensor Nodes. IEEE Transactions on Biomedical Engineering, 58, 2456-2466. http://dx.doi.org/10.1109/TBME.2011.2156795

[6] Noury, N., Herve, T., Rialle, V., et al. (2000) Monitoring Behavior in Home Using a Smart Fall Sensor and Position Sensors. 1st Annual International Conference on Microtechnologies in Medicine and Biology (ICMMB 00), 607-610. http://dx.doi.org/10.1109/MMB.2000.893857

[7] Zatout, Y. and Campo, E. (2012) Using Wireless Technologies for Healthcare Monitoring at Home: A Survey. IEEE 14th International Conference on e-Health Networking, Applications and Services (HealthCom 12), 383-386. http://dx.doi.org/10.1109/HealthCom.2012.6379443

[8] Pomponio, L., Le Goc, M., Pascual, E. and Anfosso, A. (2011) Resident's Activity at Different Abstraction Levels: Proposition of a General Theoretical Framework. IEEE 6th International Conference on Intelligent Data Acquisition and Advanced Computing Systems (IDAACS 11), 540-545. http://dx.doi.org/10.1109/IDAACS.2011.6072825

[9] van den Bossche, A., Val, T. and Campo, E. (2007) Prototyping and Performance Analysis of a QoS MAC Layer for Industrial Wireless Network. 7th International Conference on Fieldbuses and Networks in Industrial and Embedded Systems (IFAC 07), 7. http://dx.doi.org/10.3182/20071107-3-FR-3907.00028

[10] Mahfoudh, S. and Minet, P. (2009) Maximization of Energy Efficiency in Wireless Ad Hoc and Sensor Networks with SERENA. Mobile Information Systems, Advances in Wireless Networks, 5, 33-52.

[11] IEEE 802.15.4 Standard (2006) Part 15.4: Wireless Medium Access Control (MAC) and Physical Layer (PHY) Specifications for Low-Rate Wireless Personal Area Networks (LR-WPANs). IEEE Standard for Information Technology, IEEE-SA Standards Board.

[12] Yoo, D.S., Park, S.S., Choi, S.S. and Park, S.H. (2008) Dynamic S-MAC Protocol for Wireless Sensor Networks Based on Network Traffic States. 14th Asia-Pacific Conference on Communications (APCC 2008), Tokyo, 14-16 October 2008, 1-5.

[13] Jeong Gil, K., Tsiftes, N., Dunkels, A. and Terzis, A. (2012) Pragmatic Low-Power Interoperability: ContikiMAC vs 
TinyOS LPL. 9th Annual IEEE Communications Society Conference on Sensor, Mesh and Ad Hoc Communications and Networks (SECON 2012), Seoul, 18-21 June 2012, 94-96. http://dx.doi.org/10.1109/SECON.2012.6276358

[14] Mihai, G., Alina, D.A. and Ion, B. (2010) Performance Analysis on T-MAC Protocol over a Body Area Network. 3rd International Symposium on Electrical and Electronics Engineering (ISEEE 2010), Galati, 16-18 September 2010, 224-227. http://dx.doi.org/10.1109/ISEEE.2010.5628510

[15] Yang, O. and Heinzelman, W.B. (2012) Modeling and Performance Analysis for Duty-Cycled MAC Protocols with Applications to S-MAC and X-MAC. IEEE Transactions on Mobile Computing, 11, 905-921. http://dx.doi.org/10.1109/TMC.2011.121

[16] ZigBee-Alliance. http://www.zigbee.org

[17] Koubaa, A., Cunha, A. and Alves, M. (2007) A Time Division Beacon Scheduling Mechanism for IEEE 802.15.4/ Zigbee Cluster-Tree Wireless Sensor Networks. 19th Euromicro Conference on Real-Time Systems (ECRTS 2007), Pisa, 4-6 July 2007, 125-135. http://dx.doi.org/10.1109/ECRTS.2007.82

[18] Koubâa, A., Alves, M., Attia, M. and Van Nieuwenhuyse, A. (2007) Collision-Free Beacon Scheduling Mechanisms for IEEE 802.15.4/Zigbee Cluster-Tree Wireless Sensor Networks. 7th International Workshop on Applications and Services in Wireless Networks (ASWN 2007), Santander, 24-25 May 2007.

[19] Dang, T., Devic, C., Livolant, E., Van Den Bossche, A. and Val, T. (2008) OCARI: Optimization of Communication for Ad Hoc Reliable Industrial Networks. 6th IEEE International Conference on Industrial Informatics (INDIN 2008), Daejeon, 13-16 July 2008, 688-693. http://dx.doi.org/10.1109/INDIN.2008.4618189

[20] Alagha, K., Chalhoub, G., Guitton, A., Livolant, E., Mahfoudh, S., Minet, P., Misson, M., Rahme, J., Val, T. and van den Bossche, A. (2009) Cross-Layering in an Industrial Wireless Sensor Network: Case Study of OCARI. Journal of Networks, 4, 411-420. http://dx.doi.org/10.4304/jnw.4.6.411-420

[21] Muthukumaran, P.S., de Paz, R., Špinar, R. and Pesch, D. (2010) MeshMAC: Enabling Mesh Networking over IEEE 802.15.4 through Distributed Beacon Scheduling. AD HOC Networks, 28, 561-575. http://dx.doi.org/10.1007/978-3-642-11723-7 38

[22] Carballido Villaverde, B., De Paz Alberola, R., Rea, S. and Pesch, D. (2010) Experimental Evaluation of Beacon Scheduling Mechanisms for Multihop IEEE 802.15.4 Wireless Sensor Networks. 4th Conference on Sensor Technologies and Applications (SENSORCOMM 2010), Venice, 18-25 July 2010, 226-231. http://dx.doi.org/10.1109/SENSORCOMM.2010.42

[23] de Paz Alberola, R., Villaverde, B.C. and Pesch, D. (2011) Distributed Duty Cycle Management for IEEE 802.15.4 Beacon-Enabled Wireless Mesh Sensor Networks. IEEE 8th International Conference on Mobile Adhoc and Sensor Systems (MASS 2011), Valencia, 17-22 October 2011, 721-726. http://dx.doi.org/10.1109/MASS.2011.78

[24] Lu, J., van den Bossche, A. and Campo, E. (2011) An Adaptive and Distributed Collision-Free MAC Protocol for Wireless Personal Area Networks. 6th International Symposium on Intelligent Systems Techniques for Ad hoc and Wireless Sensor Networks (IST-AWSN 11), Niagara Falls, 19-21 September 2011, 798-803. http://dx.doi.org/10.1016/j.procs.2011.07.109

[25] Lu, J., van den Bossche, A. and Campo, E. (2012) Improving Robustness and Flexibility of MAC Layer for Guaranteed QoS Indoor Monitoring in Wireless Mesh Sensor Networks. 3rd International Conference on Wireless Communications in Unusual and Confined Areas (ICWCUCA 12), Clermont Ferrand, 28-30 August 2012, 1-6. http://dx.doi.org/10.1109/ICWCUCA.2012.6402491

[26] www.open-zb.net/wpan_simulator.php

[27] Fourty, N., van den Bossche, A. and Val, T. (2012) An Advanced Study of Energy Consumption in an IEEE 802.15.4 Based Network: Everything but the Truth on 802.15.4 Node Lifetime. Elsevier, Computer Communications, 35, 17591767.

[28] Van den Bossche, A. and Val, T. (2013) WiNo: Une plateforme d'émulation et de prototypage rapide pour l'ingénierie des protocoles en réseaux de capteurs sans fil. UbiMob, Nancy.

[29] http://www.daintree.net/sna/sna.php

[30] http://mi.iut-blagnac.fr

[31] Campo, E., Daran, X. and Redon, L. (2011) A Smart Home between Techniques Sciences and Human Sciences. Une maison intelligente au carrefour des sciences technologiques et des sciences humaines. 2nd Conference on Accessibility and Assistive Systems for Persons in Disabled Situation (ASSISTH 11), 33-42.

[32] http://irit.fr/ Adrien.Van-Den-Bossche/adcf_application_demonstration_video_v2.flv

[33] http://lab.iut-blagnac.fr/adcf/

[34] http://espeak.sourceforge.net/ 
Scientific Research Publishing (SCIRP) is one of the largest Open Access journal publishers. It is currently publishing more than 200 open access, online, peer-reviewed journals covering a wide range of academic disciplines. SCIRP serves the worldwide academic communities and contributes to the progress and application of science with its publication.

Other selected journals from SCIRP are listed as below. Submit your manuscript to us via either submit@scirp.org or Online Submission Portal.
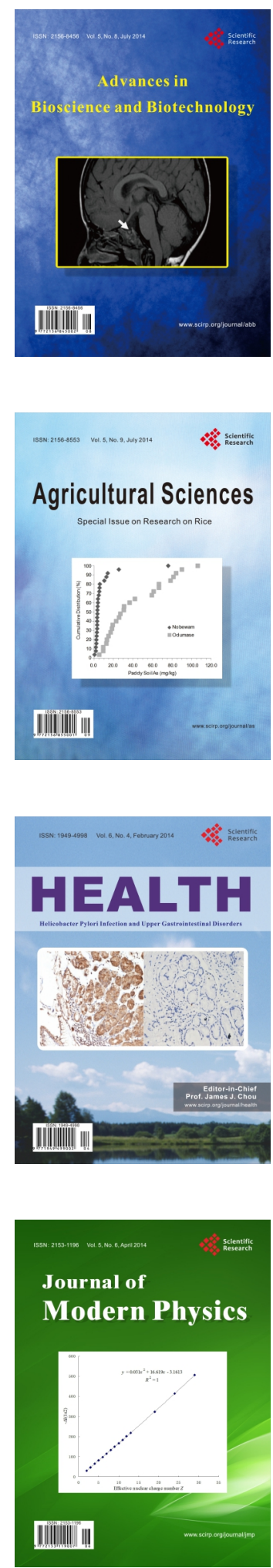
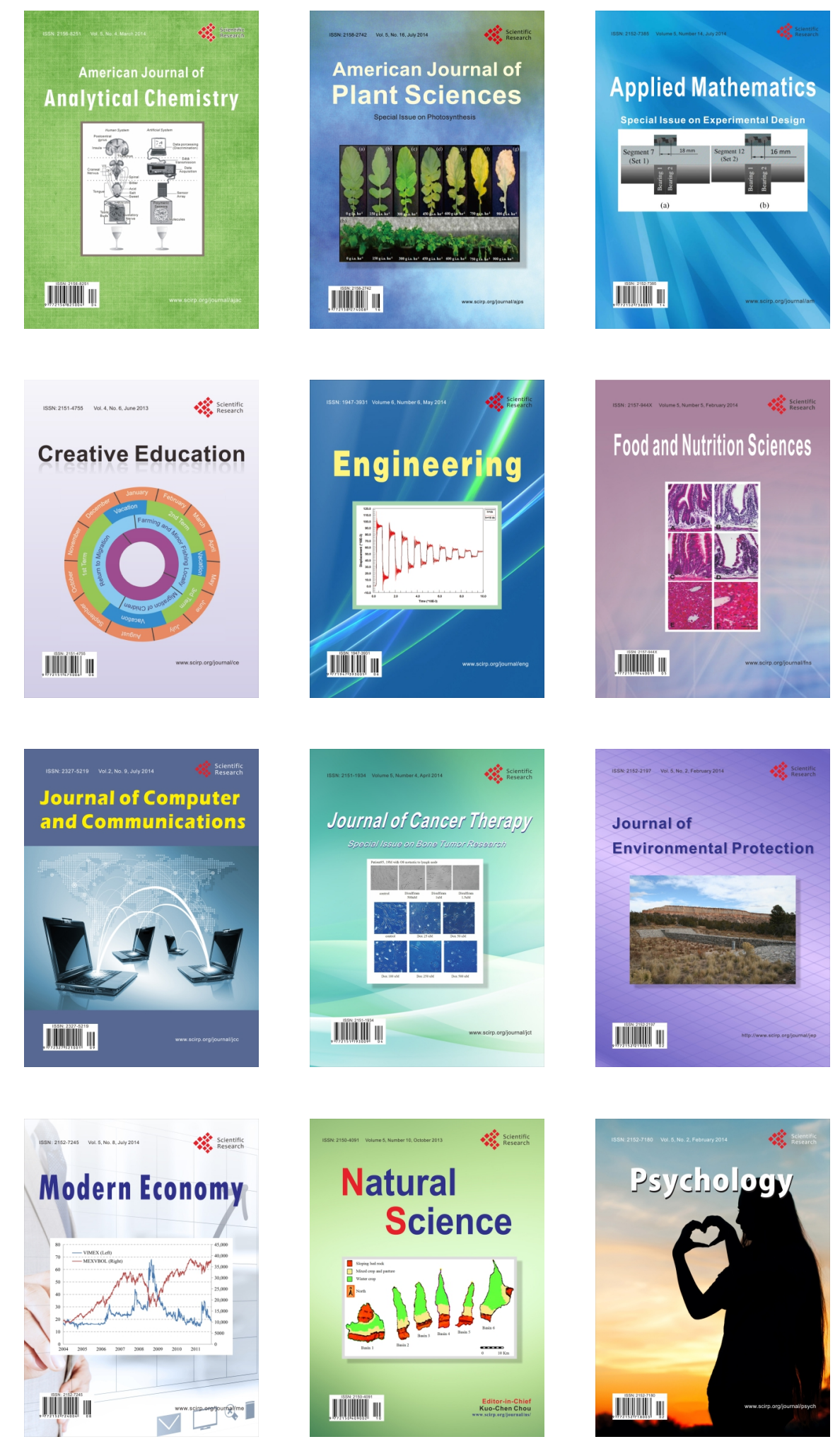